Series A

I. MATHEMATICA

408

\title{
ON THE DISCRETIZATION ERROR OF THE DIRICHLET PROBLEM IN A PLANE REGION WITH CORNERS
}

BY

PENTTI LAASONEN

H E L S I K I 1967

S U O M A L A INEN TIEDEAKA TEM I A

doi:10.5186/aasfm.1967.408 
Delivered to the Printer 20 June 1967 


\section{Introduction}

The classical Dirichlet problem in the plane is: Given a bounded plane region with prescribed values on the boundary, determine the function which is harmonic inside the region and obtains these boundary values. When encountered in practice, this problem has been subordinate to certain restrictions on the regularity of the boundary and the boundary values: they have been piecewise analytic. Hence it does not seem to be too restrictive for the applicability of the results if an investigation of the error of specific practical approximate solutions is subject to the same limitation. On the other hand, particularly considering the usability, only piecewise analyticity has to be required, because the common practical situation almost without exceptions - is that the boundary curve is composed of several analytical arcs on which piecewise analytic boundary values are prescribed. Therefore corners where different arcs meet forming some interior angles $\alpha \pi$ with $\alpha<,=$ or $>1$ have to be taken into consideration. This is important to notice, since specifically the corners originate the singularities whose presence is significant when estimating the precision and serviceability of an approximation method.

When finite difference approximation on a square net with mesh size $h$ and with the simplest five point difference operator is used the crucial effect of the corners has been previously noticed. It was shown ten years ago [5] that if the pointwise error is to be represented in the form $O\left(h^{\varkappa}\right)$, then the exponent $x$ is decisively dependent on the greatest angle. More precisely, if the greatest angle is $\alpha \pi$ and no requirement on the continuity of the boundary values at the corner points is presumed then the $x$ to be used in the estimate is

$$
\varkappa= \begin{cases}2, & \text { if } \quad \alpha \leq 1 / 2 \\ 1 / \alpha-\varepsilon, \varepsilon>0, & \text { if } \quad \alpha>1 / 2 .\end{cases}
$$

Though these results were given as valid for any subdomain excluding the

This research was supported in part by the National Science Foundation under Grant NSF-GP-4921 and the Office of Naval Research under Grant NONR(G) 00030-65. 
corner points, the material outcome of the proof can be given in the more informative estimate for the discretization error at a point $P$ :

$$
\left|\delta_{h}\right| \leq K \Sigma\left(\frac{h}{r_{v}}\right)^{x_{\nu}}
$$

where $K$ is a constant, independent of $h$ and $P$, the summation contains terms corresponding to all corners, $r_{\nu}$ 's are the respective distances of $P$ from the corner points, and $\varkappa_{\nu}$ 's depend by (1.1) on the corresponding corner angles. The estimate is valid for any point which has all its $r_{v}$ values $>k h$, where $k$ is a constant independent of $h$. When the result is represented in this form, one immediately notices that as a matter of fact the restriction on location of $P$ is unnecessary, because in any $k h$-neighborhood of a corner the quotient $\frac{h}{r}$ is bounded away from 0 and on the other hand $\left|\delta_{h}\right|$ is bounded by the maximum variation of the boundary values.

For piecewise analytic boundary values which are continuous at the corners it was shown that

$$
x=2 \text {, if } \alpha<1 \text {. }
$$

More precisely, the proof implied the existence of a constant $K$ such that

$$
\left|\delta_{h}\right| \leq K \Sigma \frac{h^{2}}{r_{\nu}}
$$

is valid outside some $k h$-neighborhood of the corner points. Actually, again this restriction is unnecessary, because the proof contains a statement that $\delta_{h}=O(r)$ and in the excluded neighborhood this, of course, may be replaced by $O\left(\frac{h^{2}}{r}\right)$.

In another paper [6] the following conjecture on the behavior of the discrete solution in the corner was made:

$$
f_{h}-f_{0}=O\left(r^{1 / \alpha}\right),
$$

where $f_{0}$ is the value of $f$ at the corner point. According to the paper, this should imply for corners with continuous boundary values the pointwise estimate

$$
\delta_{h}=O\left(h^{\varkappa}\right), \varkappa=2 / \alpha-\varepsilon, \varepsilon>0, \text { for } \alpha>1 .
$$

Two years ago Hubbard in a symposium talk [3], which was partially based on common work with Bramble and Zlamal [1], gave new results on 
the effect of singularities on the discretization error. One of the implications is that the error in the case of continuous boundary values is

$$
\left|\delta_{h}\right| \leq K h^{*}, x=1 / \alpha-\varepsilon, \varepsilon>0, \text { for } 1 / 2 \leq \alpha \leq 2
$$

uniformly in the region. As far as the dependence on $h$ is concerned for obtuse angles this result is weaker than the previous result (1.2) (only as good as (1.1), valid for discontinuous boundary values) and for concave angles weaker than the conjectured expression (1.4). However, by explicitly stating for this weaker expression its independence of $r$ it immediately leads to the conjectured behavior (1.3) (only $r^{\varkappa}$ with $x<1 / \alpha$ replacing $\left.r^{1 / \alpha}\right)$ and so enables one to prove the more precise result

$$
\left|\delta_{h}\right| \leq K h^{2 / \alpha-2 \varepsilon} r^{-1 / \alpha+\varepsilon}, \varepsilon>0, \text { for } \alpha \geq 1 .
$$

For two reasons in the following, where the main goal is proving of this estimate, however, Hubbard's result has not been used, though this would have shortened the longest section, the third, to a few statements. For one thing, now the proof is accomplished consistently with those in [5], solely by modifying Gerschgorin's method, without need to resort to results obtained by discrete Green's functions. More material, however, has been the wish to avoid those restrictions on the placement of the mesh which burden the results in [1] and [3]. After the preliminaries in section 2 and the auxiliary theorem in section 3 the main result is proved in section 4 , whereafter a concluding section summarizes the outcome of the previous and the new results.

\section{Preliminaries}

Since the result of this paper actually completes the previous paper [5] by discussing the case with continuous boundary values and greatest angle reflexive, which earlier had not been settled, similar notations are used: $D$ is a region in the plane, $B$ its boundary composed of a finite number of arcs, each of these being analytic including the endpoints; $D_{h}$ denotes the net of those mesh points of the square grid of the plane which lie in $D$ or on $B ; f$ is a given boundary function, piecewise analytic in the local arc length of $B ; f(x, y)$ is the solution of the Dirichlet problem corresponding to $D$ and $f ; f_{h}(x, y)$ is the discrete approximation of $f(x, y)$, defined as the solution of the difference equation

$$
\begin{gathered}
\Delta_{h} f_{h}(x, y)=h^{-2}\left[f_{h}(x+h, y)+f_{h}(x, y+h)+f_{h}(x-h, y)+\right. \\
\left.f_{h}(x, y-h)-4 f_{h}(x, y)\right]=0
\end{gathered}
$$

when the boundary conditions have been taken into account in a manner 
to be described hereinafter; $\delta_{h}(x, y)$ is the discretization error, i.e., the difference of $f_{h}(x, y)$ and $f(x, y)$ at points of $D_{h}$.

An equation (2.1) corresponds to each regular interior point of $D_{h}$, i.e., to those points whose four neighbor points also belong to $D_{h}$. The remaining irregular points in $D_{h}$ are so close to the boundary that at least one of the neighbor mesh points should lie outside the region. To have the necessary equations for these points, too, several methods have been applied. In the paper [5], to obtain the maximum accuracy, the linear extrapolation principle proposed by Collatz [2], was used: If the mesh line through an irregular mesh point $P(x, y)$ cuts the boundary $B$ for instance at $(x-\alpha h, y), 0<\alpha<1$, then take the neighbor mesh point at the opposite side $(x+h, y)$ (or, if this is outside the region, the boundary point $(x+\beta h, y), 0<\beta<1)$, and require the linearity of $f_{h}$ on these three consecutive points, of which at least one carries a prescribed value of $f_{h}$.

Another means to supply the irregular points with corresponding linear equations is the adoption of the irregular difference schemes for the Laplacian (see, e.g., [3]). The so-called Shortley-Weller scheme uses for instance in the above described case the linear combination

$$
\frac{2}{(\alpha+\beta) h^{2}}\left[\frac{1}{\alpha} f_{h}(x-\alpha h, y)-\left(\frac{1}{\alpha}+\frac{1}{\beta}\right) f_{h}(x, y)+\frac{1}{\beta} f_{h}(x+\beta h, y)\right]
$$

to replace the derivative $f_{x x}(x, y)$ and in a symmetrized form the combination

$$
\frac{1}{h^{2}}\left[\frac{1}{\alpha} f_{h}(x-\alpha h, y)-\left(\frac{1}{\alpha}+\frac{1}{\beta}\right) f_{h}(x, y)+\frac{1}{\beta} f_{h}(x+\beta h, y)\right] .
$$

In the following it will be assumed that any of these three methods is used.

Expansion of $f(x, y)$ in the first terms in its Taylor's series at an irregular mesh point $P$ shows that if Collatz' method of boundary adjustment is applied then

$$
\left|\delta_{h}(P)\right| \leq \frac{1}{2}\left|\delta_{h}(Q)\right|+h^{2} M^{(2)},
$$

where $Q$ is the neighbour mesh point used in the linear extrapolation and $M^{(v)}$ is some upper bound of the respective $v$ :th order derivative of $f$ on the three point mesh line segment. In case of either of the two five point crosses on an irregular mesh point $P$ the corresponding estimate is

$$
\left|\delta_{h}(P)\right| \leq \frac{1}{4} \Sigma\left|\delta_{h}\left(Q^{(\mu)}\right)\right|+h^{2} M^{(2)},
$$


where the sum contains at most three terms corresponding to all neighbor nodes $Q^{(\mu)}$ of $P$ belonging to $D_{h}$ and $M^{(2)}$ is an upper bound for the second derivatives $f_{x x}$ and $f_{y y}$ on the four legs of the mesh line cross at $P$.

\section{The behavior of the discretized solution in a reflexive corner}

The first derivatives of the solution of the Dirichlet problem in a corner $\alpha \pi>\pi$ depend on the distance $r$ from the corner point by a function $O\left(r^{1 / \alpha-1}\right)$ (see e.g., [4]), which implies the behavior $O\left(r^{1 / \alpha}\right)$ of the variation of $f$. In [6] the conjecture (1.3) was made that this is true also for the discretized solution $f_{h}$. In the following a theorem will be proved whose limiting contents (for $\varepsilon \rightarrow 0$ ) would confirm this conjecture.

Theorem 1. If the plane region has a corner at $O$ with the interior angle $\alpha \pi, \alpha \geq 1$, between two analytic arcs, where the analytic boundary values on both sides have the same limit $f_{0}$ at the corner point, then there exists for each $\varepsilon>0$ a constant $K$ such that the discrete approximations $f_{h}$ of the Dirichlet problem solution have a variation

$$
\left|f_{h}-f_{0}\right|<K r^{1 / \alpha-\varepsilon}, \quad \varepsilon>0
$$

where $r$ is the distance from the corner point; however, if $r<h / 2$, then the right hand side must be replaced by $K h^{1 / \alpha-\varepsilon}$.

Proof. Obviously it does not essentially restrict the general validity of the proof when in following the value $f_{0}$ is normed to be $f_{0}=0$.

Introduce a local polar coordinate system $(r, \varphi)$ with $r=0$ at the corner point $O$ and $\varphi=0$ the bisector of the interior corner angle. The analyticity of the corner sides implies, for any $\varepsilon>0$, the existence of positive constants $\alpha_{1}$, with

$$
1 / \alpha-\varepsilon<1 / \alpha_{1}<1 / \alpha,
$$

and $r_{1}$ such that up to the distance $r_{1}$ the region is located totally in the angle

$$
|\varphi| \leq \alpha_{1} \pi / 2 .
$$

$r_{1}$ may be assumed to be so small that no other corners belong to the circle on $O$ with radius $r_{1}$. Choose any positive constants $\bar{\alpha}_{1}$ and $\varkappa$, subject to the inequalities

$$
1 / \alpha-\varepsilon \leq \varkappa<1 / \bar{\alpha}_{1}<1 / \alpha_{1} .
$$

Consider first the strictly concave angles $\alpha \pi>\pi$; at the end of this section a remark will be included to substantiate the correctness of the proof for the limit case $\alpha=1$. 
The function $f_{h}$ will be compared with the function $K F(P)$ where

$$
\begin{gathered}
F(P)=F(r, \varphi)=r^{*}\left[\cos \varkappa \varphi-c_{1}\right] \\
c_{1}=\cos \left(\varkappa \bar{\alpha}_{1} \pi / 2\right)>0
\end{gathered}
$$

and $K$ will be determined later. Let $k$ be another positive constant which also will be determined later. When only mesh constants $h<r_{1} / k$ are considered, then there exists for each $h$ an annulus with the fixed outer radius $r_{1}$ and the $h$-dependent inner radius $k h$. The mesh points in the intersection of this annulus and $D$ will be denoted by $D_{h, k}$ and in particular the set of the regular interior points by $D_{h, k}^{\prime}$ and the set of the irregular ones by $D_{h, k}^{\prime \prime}$. By the definition of the parameters $\alpha_{1}, \bar{\alpha}_{1}, \varkappa$ and $r_{1}$ we have

$$
\begin{aligned}
F(P) & \geq b r^{*}, & P \in D_{h, k} \\
b & =\cos \left(\varkappa \alpha_{1} \pi / 2\right)-\cos \left(\varkappa \bar{\alpha}_{1} \pi / 2\right)>0 . &
\end{aligned}
$$

The discrete Laplacian of $F$ in $D_{h, k}^{\prime}$ is

$$
\begin{aligned}
\Delta_{h} F & =\Delta F+h^{2} O\left(F^{(4)}\right) \\
& =-c_{1} \varkappa^{2} r^{\varkappa-2}+h^{2} O\left(r^{\varkappa-4}\right) \\
& =-c_{1} \varkappa^{2} r^{\varkappa-2}\left[1+O\left(\frac{h^{2}}{r^{2}}\right)\right]
\end{aligned}
$$

where $F^{(i)}$ stands for an upper bound for the absolute values of the $i$ :th derivatives of $F$ on the mesh line cross in question. The last expression implies the existence of a positive constant $k_{1}$ such that if $k \geq k_{1}$, then

$$
\Delta_{h} F(P)<-\frac{1}{2} c_{1} \varkappa^{2} r^{*-2}, \quad P \in D_{h, k}^{\prime} .
$$

Later on $k$ will be submitted to a second similar condition.

Besides $f_{h}$, which is defined at all interior points $D_{h}$, another discretized function, $f_{h}^{*}$, will be considered which is defined on $D_{h, k}$. For this purpose $D_{h, k}$ must be completed by the necessary boundary points, which are either mesh points outside the circle $r=r_{1}$, mesh points inside the circle $r=k h$, or points of $B_{h}$. On all these boundary points the value of $f$ is also given for the function $f_{h}^{*}$. Then the requirement $\Delta_{h} f_{h}^{*}=0$ defines $f_{h}^{*}$ uniquely in the interior points $D_{h, k}$. Let $\delta_{h}^{*}=f_{h}^{*}-f$. Its discrete Laplacian on $D_{h, k}^{\prime}$ is

$$
\Delta_{h} \delta_{h}^{*}=-\Delta_{h} f=-\frac{1}{6} h^{2} M^{(4)}=O\left(h^{2} r^{1 / \alpha-4}\right)=\frac{h^{2}}{r^{2}} O\left(r^{\kappa-2}\right) .
$$


Hence for a suitably chosen positive constant $K^{\prime}$

$$
\left|\Delta_{h} \delta_{h}^{*}\right|<K^{\prime} r^{*-2}
$$

on $D_{h, k}^{\prime}$. If $K$ is chosen to satisfy the inequality

$$
\frac{1}{2} c_{1} \varkappa^{2} K>K^{\prime}
$$

then by (3.2) the discrete Laplacians of

$$
K F+\delta_{h}^{*} \quad \text { and } \quad K F-\delta_{h}^{*}
$$

are negative on $D_{h, k}^{\prime}$.

Next an argument by contradiction will prove that if $k$ has been chosen great enough then there exist constants $K$ so large that for any net these functions are positive on $D_{h, k}^{\prime \prime}$, at least for sufficiently small mesh constants $h$. The opposite assumption implies the existence of a monotonically decreasing sequence of mesh constants, $h_{1}, h_{2}, \ldots$, such that for any $h_{v}$ there exist a set $D_{h_{\nu}, k}$, a point $P_{v}$ on the subset $D_{h_{\nu}, k}^{\prime \prime}$ of $D_{h_{v}, k}$, and a constant $K_{v}$ such that the two functions (3.4) for $K=K_{v}$ are nonnegative on $D_{h_{\nu}, k}^{\prime \prime}$, and at $P_{v}$ one of them vanishes, and the factors $K_{\nu}$, all satisfying $(3.3)$, increase indefinitely with $v \rightarrow \infty$. These functions then are also non-negative on $D_{h_{\nu}, k}^{\prime}$.

Let $Q_{\nu}^{(u)}$ be the neighbor nodes of $P_{v}$, whereby their number is either 1,2 or 3 . From the relations

$$
\begin{aligned}
& \left|\delta_{h_{\nu}}^{*}\left(P_{\nu}\right)\right|=K_{\nu} F\left(P_{\nu}\right) \\
& \left|\delta_{h_{\nu}}^{*}\left(Q_{\nu}^{(u)}\right)\right| \leq K_{\nu} F\left(Q_{\nu}^{(u)}\right)
\end{aligned}
$$

and from the equations (2.2) or (2.3) we deduce the inequalities

$$
K_{v}\left[F\left(P_{v}\right)-\frac{1}{2} F\left(Q_{v}\right)\right] \leq h^{2} M^{(2)},
$$

in the first type boundary adjustment, and

$$
K_{\nu}\left[F\left(P_{\nu}\right)-\frac{1}{4} \Sigma F\left(Q_{\nu}^{(\mu)}\right)\right] \leq h^{2} M^{(2)}
$$

in the second and third type boundary adjustments, respectively. Since the points $Q_{v}$ have distances $\leq h$ from $P_{v}$, we have

$$
\left|F\left(Q_{\nu}^{(\mu)}\right)-F\left(P_{v}\right)\right| \leq h F^{(1)}=h O\left(r^{\nu-1}\right) .
$$

This leads, by the above inequalities, to the relation 


$$
K_{v}\left[\frac{1}{4} F\left(P_{v}\right)+h O\left(r^{\varkappa-1}\right)\right] \leq h^{2} M^{(2)},
$$

or in view of the lower bound (3.1),

$$
\frac{1}{4} K_{\nu} F\left(P_{\nu}\right)\left[1+O\left(\frac{h}{r}\right)\right] \leq h^{2} M^{(2)} .
$$

The expression in brackets implies the existence of a constant $k_{2}$ such that if $k>k_{2}$, then for $P_{v}$ belonging to $D_{h, k}$, this bracket is greater than a positive constant. This leads, however, to a contradiction with the properties (3.1) for $F, M^{(2)}=O\left(r^{1 / \alpha-2}\right)$, and with the possibility of $K_{v}$ increasing indefinitely.

The existence of such a constant $K$ thus assures that the functions $K F \pm \delta_{h}^{*}$ are positive on $D_{h, k}^{\prime \prime}$ and have negative Laplacians on $D_{h, k}^{\prime}$, irrespective of $h$ which however has to be sufficiently small; thus the inequality

$$
\left|\delta_{h}^{*}\right|<K F
$$

is found to be valid in $D_{h, k}$. Considering the behavior $f=O\left(r^{1 / \alpha}\right)=O\left(r^{*}\right)$ of $f$ this implies the existence of a constant $\bar{K}$ such that

$$
\left|f_{h}^{*}\right| \leq|f|+\left|\delta_{h}^{*}\right|<\bar{K} r^{*}
$$

is true for any sufficiently small $h$ in $D_{h, k}$.

Now it will be proved that a similar bound is valid for an extension function $\bar{f}_{h}^{*}$ of $f_{h}^{*}$ to mesh points with $r<k h$. Assume that $\bar{K}$ is so large that

$$
|f|<\bar{K} h^{*}
$$

in the intersection of $D_{h}+B_{h}$ and the circle on $O$ with radius $k h$, and

$$
\left|f_{h}^{*}\right|<\bar{K} h^{*}
$$

in the intersection of $D_{h, k}$ and the circle on $O$ with radius $(k+1) h$. The existence of a constant subject to the former condition is motivated by the behavior of $f$ in the corner, mentioned above, whereas the second condition is a direct application of (3.5). We will now define $\bar{f}_{h}^{*}$ and prove the existence of a constant $C$, dependent only on the number $n$ of mesh points in the intersection of $D_{h}+B_{h}$ and the circle on $O$ with radius $k h$, such that

$$
\left|\bar{f}_{h}^{*}\right|<C \overline{\bar{K}} h^{*} \text {. }
$$

To this end we will join to $D_{h, k}$ those points $P_{1}, P_{2}, \ldots, P_{n}$ with $r<k h$ which were previously excluded from $D_{h, k}$ and which are not among its later associated boundary points, one at a time; first all mesh points, then the intersection points of mesh lines and $B$. Let the value 
of $f$ be given at the point $P_{j}$ at the time the point is joined to the set, whereafter the necessary revaluation will be performed in the entire point set $D_{h, k}+P_{1}+P_{2}+\ldots+P_{j}$ in order to have the discrete Laplacian vanish at each interior point. The new function may be called $f_{h, j}$, with $f_{h, 0}=f_{h}^{*}$; define $f_{h, n}=\bar{f}_{h}^{*}$. The difference of $f_{h, j}$ and $f_{h, j-1}$ will now be evaluated.

The adjunction of $P_{j}$ to $D_{h, k}+P_{1}+\ldots+P_{j-1}$ may change the status of some of its neighbor mesh points in the previous set from boundary points to interior points. If none of them changes status, then $f_{h, j}=f_{h, j-1}$. Let now $Q$ be a neighbor mesh point where such a change happens. In general the value $f\left(P_{j}\right)$ does not suit to the previous values $f_{h, j-1}$ at $Q$ and its other three neighbors to make the discrete Laplacian at $Q$ vanish. If $M_{j-1}$ is any upper bound for $f_{h, j-1}$ at $Q$ and at those three previous neighbors, then such a fitting value at $P_{j}$ would be absolutely $\leq 7 M_{j-1}$, thus at most $8 M_{j-1}$ different from $f\left(P_{i}\right)$, if $M_{j-1}$ is also an upper bound for $|f|$ in $r<k h$. Therefore the change from $f_{h, j-1}$ to $f_{h, j}$ must be absolutely less than $8 M_{j-1}$ everywhere on $D_{h, k}+P_{1} \ldots+P_{j-1}$. If $M_{j-1}$ is defined as an upper bound for $f_{h, j}$ not only at $Q$ and its neighbors but at all points in $r<(k+1) h$ where this function is defined and for $f$ in $r<k h$, then

$$
M_{j}<9 M_{j-1} \text {. }
$$

This recursion inequality shows that $C=9^{n}$ is acceptable. Since the number $n$ has an upper bound which is determined solely by $k$, the existence of a constant $K^{*}$ has been shown such that

$$
\left|\bar{f}_{h}^{*}\right|<K^{*} h^{*}
$$

is valid at mesh points with $r<k h$. In this inequality the power $h^{*}$ may be replaced by $r^{*}$ at all points with $r \geq h$ or even at all points with $r \geq a h$, with $a>0$, if the factor $K^{*}$ is modified correspondingly. By choosing $a<\frac{1}{2}$ the rule hence is obtained that this upper bound is $K r^{*}$ for all points eventually with one exception, the point lying closest to the corner point; for this point the bound is $K h^{*}$. (A closer study shows that this exception is unnecessary for $\alpha<\frac{3}{2}$ ).

As to the $f_{h}$ in the circle $r \leq r_{1}$ one notices that its difference from $\bar{f}_{h}^{*}$ cannot be greater than their difference on those boundary nodes of $D_{h, k}$ which are outside this circle and where $\bar{f}_{h}^{*}$ was defined to coincide with $f$. But according to [5] this difference, the discretization error at these points, is $O\left(h^{*}\right)$ and hence does not essentially change the result. Finally the very same kind of bound is found to be valid also outside the fixed circle $r=r_{1}$ simply due to the fact that $f_{h}$ is bounded there.

If $\varepsilon<1 / \alpha$, then the choice $x=1 / \alpha-\varepsilon$ completes the proof of Theorem 1 ; in the opposite case the validity is obvious. 
If the angle, instead of being $>\pi$, is $=\pi$, only a few modifications in the above proof are necessary. The essential reason for these modifications is that in the case $\alpha=1$ the upper bounds for the $v$ th derivatives of $f$ is not $M^{(v)}=O\left(r^{1 / \alpha-v}\right)$ but $M^{(v)}=O\left(r^{1-v}|\ln r|\right)$. However, in those two cases, where such bounds have been used, these actually have been needed in the form $M^{(v)}=O\left(r^{*-v}\right)$ which now for $x<\alpha=1$ is implied by the $M^{(v)}=O\left(r^{1-v}|\ln r|\right)$. - The very same argument may be applied in the next section to establish the correctness of the result for $\alpha=1$.

\section{The discretization error}

In the following theorem only one corner is considered but the generalization to any finite number of corners is apparent.

Theorem 2. The discretization error $\delta_{h}$ in a region with its only corner of the type explained in Theorem 1 depends on the mesh constant $h$ and the distance $r$ from the corner point by

$$
\left|\delta_{h}\right|<K h^{2 / \alpha-2_{\varepsilon}} r^{-1 / \alpha+\varepsilon}, \varepsilon<0
$$

Proof. Let $\alpha_{1}, \bar{\alpha}_{1}, \varkappa_{1}, c_{1}, b$ be the same constants as in section 3 . To apply Gerschgorin's method use the majorant function

$$
G_{h}(r, \varphi)=h^{2 \varkappa}\left[r^{-\varkappa}\left(\cos \varkappa \varphi-c_{1}\right)+c_{2}\right]
$$

multiplied by a constant to be determined later; here $c_{2}$ is any constant

$$
c_{2}>\left(2+c_{1}\right) r_{\mathbf{I}}^{-*} \text {. }
$$

$G_{h}$ is obviously positive on $D+B$. By almost identical arguments with those in connection of $\Delta_{h} F$ in section 3 the following result is obtained: there exists a positive constant $k$ such that in the regular interior points $D_{h, k}^{\prime}$ of $D_{h, k}$ which is obtained from $D_{h}$ by excluding points with $r<k h$, we have

$$
\Delta_{h} G_{h}(x, y)<-\frac{1}{2} c_{1} \varkappa^{2} h^{2 \varkappa} r^{-\varkappa-2} .
$$

The discrete Laplacian of $\delta_{h}$, on the other hand, in $D_{h, k}^{\prime}$ is

$$
\Delta_{h} \delta_{h}=-\Delta_{h} f=h^{2} O\left(r^{1 / \alpha-4}\right)=h^{2 * O}\left(r^{-*-2}\right)
$$

Therefore if $K$ is sufficiently great, say $K \geq K^{\prime}$, then both $K G_{h}+\delta_{h}$ and $K G_{h}-\delta_{h}$ have negative discrete Laplacians in $D_{h, k}^{\prime}$.

Now consider these two functions on the irregular points $D_{h, k}^{\prime \prime}$. For each value of $h$ and some corresponding mesh the factor $K$ can be chosen 
so great that the functions are non-negative on $D_{h, k}^{\prime \prime}$. But how do the sufficient coefficients $K$ behave for $h \rightarrow 0$ ? Especially, is

$$
\lim _{h \rightarrow 0} \sup _{P \in D_{h}^{\prime \prime}}\left|\delta_{h}(P)\right| / G_{h}(P)
$$

finite or infinite? An argument by contradiction will now show that it is finite.

If this were not true, then a monotonically decreasing sequence of mesh constants, $h_{1}, h_{2}, \ldots$, could be chosen such that to each $h_{v}$ would correspond a set $D_{h_{v}, k}$, a point $P_{v} \in D_{h_{v}, k}^{\prime \prime}$, and a constant $K_{v} \geq K^{\prime}$, for which the functions

$$
K_{v} G_{h_{\nu}}+\delta_{h_{v}} \quad \text { and } \quad K_{\nu} G_{h_{\nu}}-\delta_{h_{v}}
$$

are non-negative on $D_{h_{\nu}, k}^{\prime \prime}$ and one of them vanishes at $P_{\nu}$ and moreover $\lim K_{v}=\infty$. From these facts and from the definition of $K^{\prime}$ it would $v \rightarrow \infty$ then follow that

$$
\begin{array}{ll}
\left|\delta_{h_{\nu}}(Q)\right| \leq K_{\nu} G_{h_{v}}(Q), & Q \in D_{h_{\nu}, k} \\
\left|\delta_{h_{v}}\left(P_{v}\right)\right|=K_{v} G_{h_{\nu}}\left(P_{\nu}\right) . &
\end{array}
$$

Let $\varrho_{v}$ be the distance of $P_{v}$ from 0 . Next it will be shown that $\lim _{\nu \rightarrow \infty} \varrho_{v} / h_{\nu}=0$. This is a consequence of estimates for both sides in (4.3). The left hand side has, by Theorem 1, an upper bound

$$
\left|\delta_{h}\left(P_{\nu}\right)\right|<K\left(\varrho_{\nu}+h\right)^{*}<K\left(1+\frac{1}{k}\right)^{*} h_{\nu}^{*}\left(\varrho_{\nu} / h_{\nu}\right)^{*}
$$

whereas the right hand side has, provided $\varrho_{\nu} \leq r_{1}$, the lower bound

$$
K_{\nu} G_{h}\left(P_{\nu}\right) \geq K_{\nu} b h_{\nu}^{*}\left(\varrho_{\nu} / h_{\nu}\right)^{-*} .
$$

This comparison gives the inequality

$$
\left(\varrho_{\nu} / h_{\nu}\right)^{2 \varkappa}>K_{\nu} \cdot \frac{b}{K}\left(1+\frac{1}{k}\right)^{-\varkappa}
$$

which implies the indefinite increase of $\varrho_{v} / h_{v}$. If $\varrho_{v}>r_{1}$ the same conclusion is evident.

Since $P_{v}$ cannot belong to the $k h$-neighborhood of $O$ it is an irregular interior point of the original set $D_{h}$ and hence either (2.2) or (2.3) is applicable there. When (4.3) is used on the left hand side and (4.2) on the right hand side, the respective inequalities

$$
K_{v}\left[G_{h_{\nu}}\left(P_{\nu}\right)-\frac{1}{2} G_{h_{\nu}}\left(Q_{\nu}\right)\right] \leq h^{2} M^{(2)}
$$




$$
\left.K_{v}\left[G_{h_{v}}\left(P_{v}\right)-\frac{1}{4} \Sigma G_{h_{v}}\left(Q_{\nu}^{(u)}\right)\right]\right] \leq h^{2} M^{(2)}
$$

are obtained, where the $Q_{v}^{\prime} \mathrm{s}$ are the respective interior neighbors of $P_{v}$. In (4.4) the left hand side bracket may be estimated on the basis of the character of $G_{h}=O\left(r^{-*}\right)$ by

$$
\begin{aligned}
G_{h_{\nu}}\left(P_{\nu}\right)-\frac{1}{2} G_{h_{\nu}}\left(Q_{v}\right) & =\frac{1}{2} G_{h}\left(P_{\nu}\right)+h^{2 \varkappa+1} O\left(r^{-\varkappa-1}\right) \\
& >\frac{1}{2} b h^{2 \varkappa} \varrho_{\nu}^{-\varkappa}\left(1+O\left(h_{\nu} / \varrho_{\nu}\right)\right),
\end{aligned}
$$

if $\varrho_{v} \leq r_{1}$, and by

$$
\begin{aligned}
G_{h_{\nu}}\left(P_{\nu}\right)-\frac{1}{2} G_{h}\left(Q_{\nu}\right) & >\frac{1}{2} h^{2 *} r_{1}^{-\varkappa}\left(1+O\left(h_{\nu} / r_{1}\right)\right) \\
& >\frac{1}{2} h^{2 \varkappa} \varrho_{\nu}^{-\varkappa}\left(1+O\left(h_{\nu} / \varrho_{\nu}\right)\right)
\end{aligned}
$$

if $\varrho_{v}>r_{1}$. The right hand side is

$$
h^{2} M^{(2)}=h^{2} O\left(\varrho_{v}^{1 / \alpha-2}\right)=h^{2 *} O\left(\varrho_{v}^{-*}\right) .
$$

so the inequality (4.4) is incompatible with the indefinite increase of $K_{v}$. Similarly the inconsistency of the inequality (4.5) where $\Sigma$ contains at most three terms is shown.

This brings the argument by contradiction of the finiteness of (4.1) to a conclusion, which proves the existence of a uniform constant $K^{\prime \prime}$ such that $K^{\prime \prime} G_{h}-\left|\delta_{h}\right|$ is positive on $D_{h, k}$. Obviously this result may be modified to assure the existence of a constant $K$ such that

$$
\left|\delta_{h}\right|<K h^{2 *} r^{-*}
$$

is valid on $D_{h, k}$. As a matter of fact, the region of validity of this estimate may be extended to the entire $D_{h}$ because in section 3 it was found that at points with $r<k h$ estimates $f f-f_{0}\left|=O\left(h^{*}\right),\right| f_{h}-f_{0} \mid=O\left(h^{*}\right)$ and hence $\left|\delta_{h}\right|=O\left(h^{*}\right)$ are true, which directly verifies (4.6) on $D_{h}-D_{h, k}$. The choice $x=1 / x-\varepsilon$ in the case $\varepsilon<1 / x$ completes the proof; in the opposite case the correctness of the claim is obvious.

\section{Some final remarks}

In the cases of discontinuous boundary values or of continuous boundary values and convex angles estimates for the discretization error, described in the first section, were derived in [5] for the Collatz type boundary value 
adjustment. However, it is quite apparent that the two other schemes applied in this paper could also have been applied there. It has already been pointed out that it is unnecessary to restrict the region of validity of the estimates to a subdomain which excludes the corner points. Therefore the following theorem, which summarizes the previous as well as the new results, is free from restrictions of that type:

Theorem 3. Under the assumptions of piecewise analyticity of boundary and boundary values described in section 1, when the simple five point difference scheme for the discrete Laplacian and any of the three boundary value adjustments described in section 2 are applied, the discretization error $\delta_{h}$ of the Dirichlet problem solution can be estimated uniformly in the region by

$$
\left|\delta_{h}\right|<K\left(\Sigma h^{\kappa} v r_{v}^{-\lambda_{v}}+h^{2}\right) ;
$$

where each corner contributes one term to the sum and $r_{v}$ is the distance from the corner point; the exponents depend on the respective corner angles $\alpha_{v} \pi$ by the rules:

for discontinuous boundary values at the corner

$$
\varkappa_{v}=\lambda_{v}=\min \left(2,1 / \alpha_{v}-\varepsilon\right),
$$

for continuous boundary values at the corner

$$
\begin{aligned}
& \varkappa_{\nu}=\min \left(2,2 / \alpha_{v}-2 \varepsilon\right), \\
& \lambda_{\nu}=\min \left(1,1 / \alpha_{v}-\varepsilon\right) ;
\end{aligned}
$$

$\varepsilon$ is any positive number and $K$ may depend on $\varepsilon$.

As to the relaxation of the requirements on the analyticity of the boundary curve and the boundary values, the following remark may be put forth. The actual significance of analyticity is its bearing on the assumption that for the $n$th derivatives $(n \leq 4)$ of the solution of the Dirichlet problem have the behavior

$$
f^{(n)}=O\left(r^{-n}\right)
$$

at corners with discontinuous boundary values and

$$
f^{(n)}= \begin{cases}O\left(r^{1-n}\right), & \alpha<1 \\ O\left(r^{1-n}|\ln r|\right)_{2} & \alpha=1 \\ O\left(r^{1 / \alpha-n}\right), & \alpha>1\end{cases}
$$

at corners with continuous boundary values. If this behavior at the corners and the continuity elsewhere is secured, and some vicinities of the corners can be covered by circular sectors with angles $(\alpha+\eta) \pi, \eta$ positive but arbitrarily small, then the essential conditions of the theorem are satisfied and the analyticity is unnecessary. 


\section{References}

[1] Bramble, J. H., Hubbard, B. E., Zlámal, M.: Discrete analogs of the Dirichlet problem with isolated singularities, J. SIAM, ser. B. (to appear).

[2] Collatz, L.: Bemerkungen zur Fehlerabschätzung für das Differenzenverfahren bei partiellen Differentialgleichungen. ZAMM 13, 56-57, (1933).

[3] Hubbard, B. E.: Remarks on the convergence in the discrete Dirichlet problem, Numerical Solution of Partial Differential Equations (ed. J. H. Bramble), Academic Press, 21-34, (1966).

[4] Laasonen, P.: On the behavior of the solution of the Dirichlet problem at analytic corners, Ann. Acad. Sci. Fenn. A I 241, 1-13 (1957).

[5] - - On the degree of convergence of discrete approximations for the solution of the Dirichlet problem, Ann. Acad. Sci. Fenn. A I 246, 1-19, (1957).

[6] - - - On the truncation error of discrete approximations to the solutions of Dirichlet problems in a domain with corners, J. Ass. Comp. Mach. 5, 1, 32-38, (1958).

Technical University

Otaniemi, Finland 